\section{The breadth of attention in old age}

\section{Stefanie Hüttermann, 1 Otmar Bock,2 Daniel Memmert 1}

1Institute of Cognitive and Team/Racket Sport Research, German Sport University Cologne; 2Institute of Physiology and Anatomy, German Sport University Cologne, Germany

\begin{abstract}
Older adults typically have more difficulties than younger ones in situations that require attention in the visual periphery, such as driving a car or riding a bicycle. Previous studies accordingly found that the breadth of attention decreases in old age when one attentiondemanding task is presented at fixation and simultaneously another one in the visual periphery. The present work evaluates the role of eye position for the observed deficit by presenting both tasks in the visual periphery (condition peripheral-peripheral) or by leaving it up to the subjects where in the visual field the tasks appear (condition free-gaze). Our data indicate that attention breadth decreases by $27 \%$ from the age of early 20 to the age of late 60 in both conditions. This outcome generalizes previous findings about age-related attention deficits to scenarios that were not explored in previous studies, yet are relevant for everyday behavior.
\end{abstract}

\section{Introduction}

Many situations of everyday life require us to spread attention across an extended area of space, e.g., when driving a vehicle, participating in team sports, or monitoring technical equipment. The performance of older adults in such situations is typically inferior to that of young adults, ${ }^{1}$ which is a troublesome perspective for our graying society. The age-related decrease of attention breadth cannot be explained by known impairments of basic visual functions such as static visual acuity, 2,3 dynamic visual acuity, ${ }^{4}$ contrast sensitivity,, 6 and peripheral visual field size, ${ }^{7}$ and therefore seems to reflect a deficit of distributed attention as such. Indeed, it has been documented that the area of space to which attention can be devoted shrinks in old age..$^{8-10}$

The breadth of attention is typically established by asking participants to perform one attention-demanding task at fixation, and simultaneously a second one in the visual periphery; eye and head movements are discouraged by presenting the peripheral task for only a brief time.11,12 The size of the visual field in which subjects respond correctly on $\geq 75 \%$ of trials is called the useful field of view (UFoV), 13,14 and is usually smaller than the field of view determined by conventional perimetry. 15,16

The purpose of our study was to find out whether the age-related shrinkage of UFoV is limited to the above scenario - one task at fixation and the other in the periphery - or rather extends to other situations as well. To this end, we selected two situations which are also relevant for everyday life: subjects fixate midbetween the two tasks in one, and are free to move their eyes as they desire in the other. In our view, the latter situation is particularly characteristic for everyday life.

We expected that the attention breadth in our study will be smaller for older adults than for young subjects, thus endorsing the general validity of the deficit reported in literature. We further expected that this deficit is particularly pronounced when subjects are free to move their eyes, since old age is associated with oculomotor problems such as reduced saccadic speed and accuracy and increased saccadic reaction times. ${ }^{17-20}$

\section{Materials and Methods}

\section{Participants}

Twelve male young adults $(22.92 \pm 2.02$ years of age) and twelve older adults (7 female, $69.00 \pm 4.69$ years of age) participated. All lived independently in the community and reported to be in good general health and to have no eye problems besides corrected vision. Subjects who wore corrective eyeglasses or contact lenses upon arrival continued to wear them during the experiments. Written informed consent was obtained from each subject prior to participation in this study. The study was carried out in accordance with the Helsinki Declaration of 1975.

\section{Materials and Procedure}

Subjects sat at a distance of approximately $1.30 \mathrm{~m}$ from a white silver projection screen of $2.80 \mathrm{~m}$ width and $2.20 \mathrm{~m}$ height (i.e., visual angle of about $94 \times 80^{\circ}$ ). Stimuli were generated with E-Prime ${ }^{\circledR}$ and consisted of two clusters, displayed symmetrically about the screen center either on the horizontal, the vertical, the right diagonal or the left diagonal meridian. The distance between the two clusters of a stimulus was $10^{\circ}, 20^{\circ}, 30^{\circ}$ or $40^{\circ}$ (i.e., clusters were $5^{\circ}, 10^{\circ}, 15^{\circ}$ or $20^{\circ}$ from the screen center). Cluster meridians and distances varied randomly between the 192 trials of each condi-
Correspondence: Stefanie Hüttermann, Institute of Cognitive and Team/Racket Sport Research, German Sport University Cologne, Am Sportpark Müngersdorf 6, 50933 Köln, Germany.

Tel. +49.221.49824312 - Fax: +49.221 .4995637 .

E-mail: s.huettermann@dshs-koeln.de

Key words: attention window, attention breadth, attention focus, aging, older adults.

Conflict of interests: the authors report no potential conflict of interests.

Received for publication: 13 June 2012. Revision received: 18 October 2012.

Accepted for publication: 18 November 2012.

This work is licensed under a Creative Commons Attribution NonCommercial 3.0 License (CC BYNC 3.0).

CC Copyright S. Hüttermann et al., 2012

Licensee PAGEPress, Italy

Ageing Research 2012; 4:e10

doi:10.4081/ar.2012.e10

tion. As an example, Figure 1 illustrates a stimulus that consists of two clusters on the right diagonal meridian (we used an experimental task developed by Stefanie Hüttermann, Daniel Memmert, Daniel Simons and Otmar Bock).

Each cluster was composed of four $9 \times 9 \mathrm{~cm}$ elements, and thus had a size of $18 \times 18 \mathrm{~cm}$. The elements had one of two forms (circle, triangle) and one of two shades (light gray, dark gray), i.e., there were four possible elements. Form and shading varied randomly from trial to trial, such that the probability of presenting zero, one, two, three or four light gray triangles in a stimulus was $20 \%$ each. The subjects' task was to report the number of light gray triangles in each cluster, a conjunction search which is known to be attention-demanding. ${ }^{21,22}$

The serial order of events is illustrated in Figure 1. Trials began with the presentation of a fixation cross in the screen center for 1000 $\mathrm{ms}$. Two circles of $8 \mathrm{~cm}$ diameter were presented as pre-cues at the locations of the subsequent stimulus clusters for $200 \mathrm{~ms}$. Following a $200 \mathrm{~ms}$ blank screen, a cluster pair appeared at the pre-cued locations for $300 \mathrm{~ms}$. Subjects then gave unspeeded verbal reports about the number of light gray triangles first in one, and then in the other cluster. The reports were rated as correct only if the numbers given for both clusters were correct.

In condition peripheral-peripheral, subjects had to fixate the cross in the screen center and watch the cluster pairs by peripheral vision. In condition free-gaze, subjects were free to direct their gaze as desired. The order of these two conditions was counterbalanced across subjects. Each session started with a warm-up 
phase of 16 trials in each condition, followed by three blocks of 64 trials in one, and by another three blocks in the other condition. Blocks were separated by rest breaks of $30 \mathrm{~s}$.

Eye position was monitored with a headmounted eye tracking system (Eye Mobile ${ }^{\circledR}$, Applied Science Laboratories, Bedford, U.S.) which maps the gaze direction onto a video image of the surroundings with a sampling rate of $30 \mathrm{~Hz}$ and a resolution of $1^{\circ}$. In condition peripheral-peripheral, trials in which subjects failed to maintain fixation were discarded ( $5 \%$ of trials in young adults, $3 \%$ of trials in older adults).

The experiment was preceded by a perimetry test. Subjects fixated straight ahead, and a single visual object was moved from the subjects' visual periphery towards the center until it was detected. Each eye was tested separately, with the other eye covered.

\section{Data analysis}

The effects of age on attention performance were analyzed by a $2 \times 2 \times 4 \times 3$ (age group [young, older] $\times$ condition [peripheral-peripheral, free-gaze $] \times$ cluster distance $\left[10^{\circ}, 20^{\circ}\right.$, $30^{\circ}, 40^{\circ}$ ] $\mathrm{x}$ meridian [horizontal, vertical, diagonal]) analysis of variance (ANOVA) with repeated measures on the last three factors. Greenhouse-Geisser procedures were used to correct for violations of sphericity assumption. The measured attention window was defined as the stimulus distance at which subjects correctly identified both simultaneously presented stimuli in $75 \%$ of trials.

\section{Results}

In the perimetry test, we found that participants could identify objects at distances up to $55.92 \pm 4.24^{\circ}$ from the fixation mark, which fits with previous data and is well beyond the range of cluster distances in our experiment. ${ }^{23}$ The perimetry data from young and older adults were not significantly different $\left(57.50 \pm 4.52^{\circ}\right.$ versus $54.33 \pm 3.42^{\circ}, F(1,22)$ $=3.743, \mathrm{P}=0.066$ ), which is again consistent with earlier findings. 13

Figure 2 illustrates the success rates of young and older subjects as a function of cluster distance, separately for condition peripheralperipheral (top graph) and free-gaze (bottom graph). The ANOVA confirmed that success rate was significantly higher for young than for older adults, $F(1,22)=149.896, \mathrm{P}<0.001, \eta^{2}=0.872$, for peripheral-peripheral than for free-gaze, $F(1,22)=53.939, \mathrm{P}<0.001, \eta^{2}=0.710$, and for small than for large cluster distances, $F(1$, 22) $=181.202, \mathrm{P}<0.001, \eta^{2}=0.892$.

ANOVA also revealed a significant effect of the meridian, $F(1,22)=10.331, \mathrm{P}<0.001$, $\eta^{2}=0.320$. This is illustrated by Figure 3 , which shows the attention window as determined by the 75\%-correct criterion: that window was largest along the horizontal, and smallest along the vertical meridian. Across all meridians and conditions, the attention window averaged $29.74 \pm 1.82^{\circ}$ cluster distance in young and $21.70 \pm 1.98^{\circ}$ in older subjects, which represents an age-related decrease of $27 \%$.

ANOVA yielded no significant interaction terms. Specifically, age $\mathrm{x}$ distance, $F(2.066$, 45.459) $=2.836, \mathrm{P}=0.067$, and age $\times$ condition $\times$ distance, $F(2.897,63.730)=0.640, \mathrm{P}=0.587$, were both non-significant.

To determine whether older adults' performance in one condition was related to their performance in the other condition, we z-transformed their data separately for each cluster distance and meridian to remove the consistent effects of stimulus location, and then calculated the Pearson correlation between conditions. The resultant value of $r=-0.09$ was not significant $(\mathrm{P}=0.286)$.

\section{Discussion}

Our study evaluated the attention breadth of young and older subjects that fixate midbetween two attention-demanding tasks (condition peripheral-peripheral), or are free to direct their gaze at either task (condition freegaze). We used the $75 \%$-correct criterion to delimit subjects' attention window and found this window to be smaller than the perimetrically defined visual field, which confirms the observations of others. ${ }^{13}$ The attention window had an ellipsoid shape and extended further into the periphery along the horizontal than along the vertical meridian. This shape reflects well the requirements of our daily life, where objects of interest are typically more widely distributed in the horizontal than in the vertical dimension.

Since condition free-gaze did not constrain eye movements and thus allowed subjects to look straight ahead as in condition peripheralperipheral, we expected that subjects' performance in free-gaze will be equal or better than in peripheral-peripheral. Actually, however, their performance turned out to be worse. A possible explanation of this surprising outcome is that stimuli in free-gaze but not in peripheralperipheral triggered reflexive saccades, ${ }^{24,25}$ which bind neural processing resources and suppress vision, ${ }^{26-29}$ and that these oculomotor demands interfered with attentive processing. This tentative interpretation could also explain the lack of a significant correlation between both conditions: eye movements possibly introduced a source of variability to free-gaze that was absent in peripheral-peripheral.

The most interesting outcome for the purposes of our study is the effect of age, i.e., the attention window of our older subjects was $27 \%$ narrower than that of young participants.

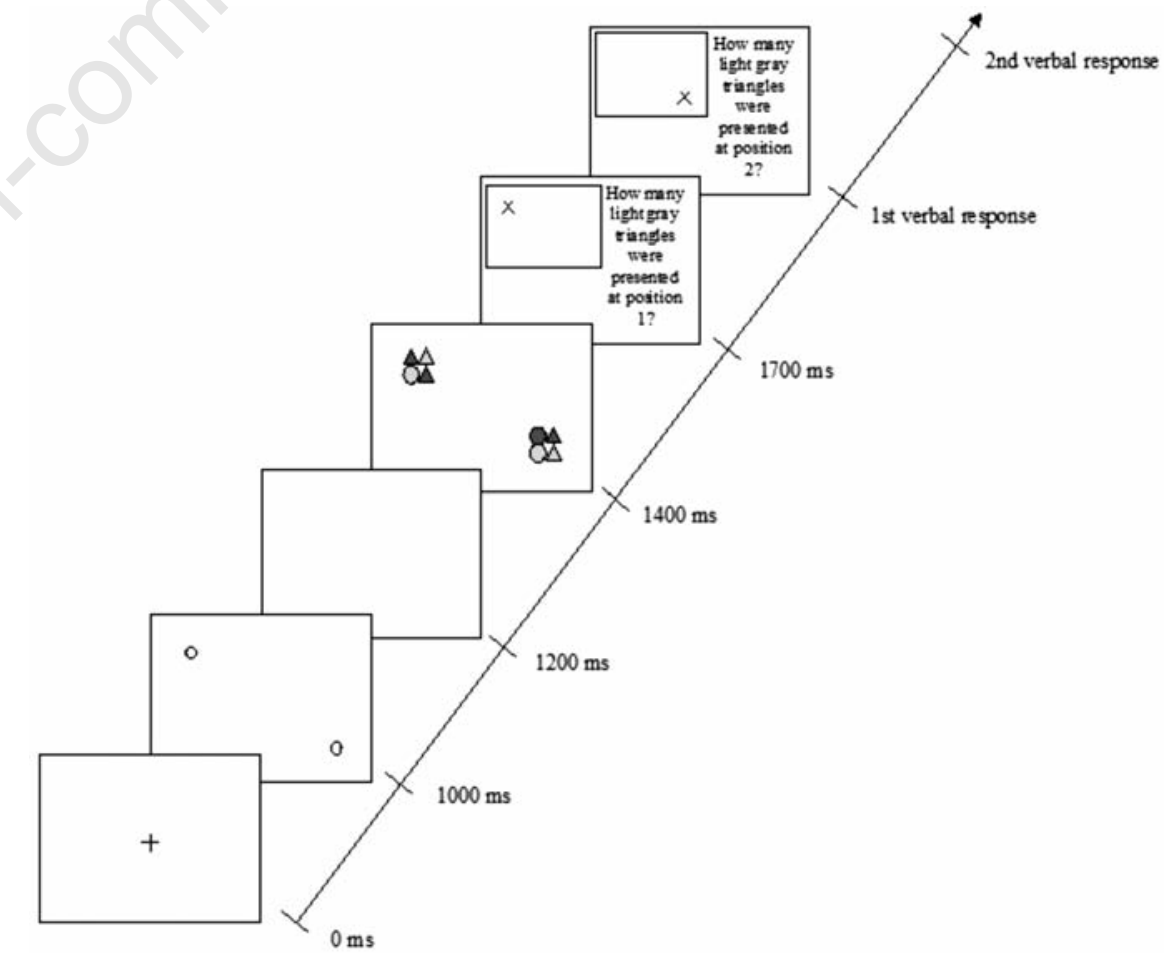

Figure 1. Sequence of events in a trial with stimuli along the right diagonal meridian. 
An age-related decrease of attention breadth has also been observed in earlier work with a somewhat different paradigm, using one task at fixation and the other in the periphery. However, the effect size was substantially larger there: a survey of published data reveals that the attention window shrank by about $67 \%$, which is more than double the amount documented in the present study.12,30,31 This discrepancy cannot be explained by age differences between studies. The mean age of older subjects was 69 both in the present and in the cited earlier studies; that of young subjects was 23 in the present and 25 or 29 in the earlier studies, that is, it could explain a larger but not a smaller effect in our data. It therefore appears that the age-related decrease of attention breadth depends on the viewing condition: it is larger when one of the two tasks is steadily fixated, possibly because a fixated task attracts attention and thus withdraws it from the periphery.

\section{Conclusions}

Summing up so far, we posit that the effects of age on attention breadth are not uniform, but rather depend on the presence or absence of eye movements and of a steadily fixated task. If so, the practical implications are obvious.
Since everyday life requires attentive processing under varying viewing conditions, training programs aimed at expanding the attention window of older persons should not be limited to one, ${ }^{32,33}$ but rather should include multiple conditions. We therefore suggest to scrutinize our assumption by further experiments and, if the outcome is confirmatory, to modify the available training programs accordingly.

A final point deserves mentioning: if the notion of a shrinking attention window is correct, the performance of older subjects should equal to that of young ones at small cluster distances and be inferior only at large distances, that is, we should yield an age $\mathrm{x}$ distance effect. In contrast to this expectation, however,

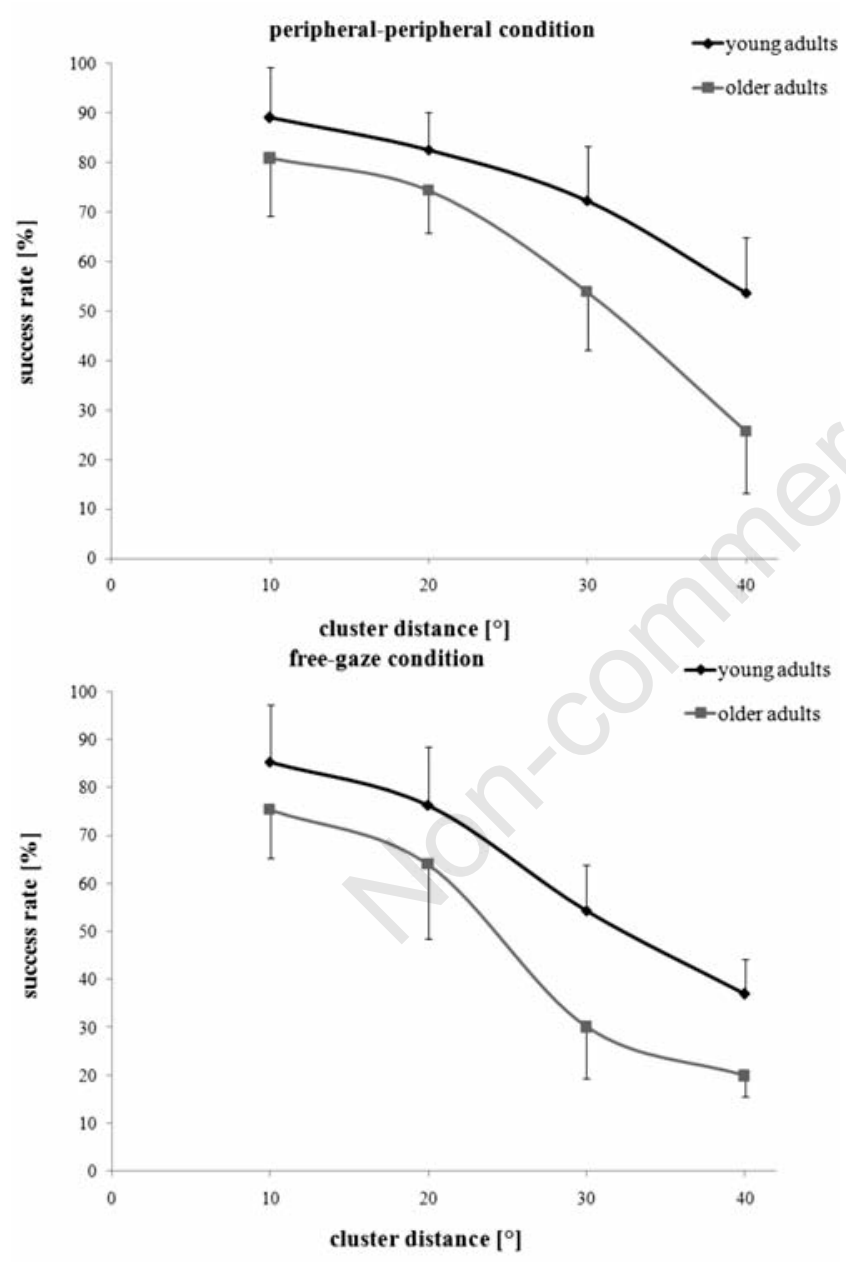

Figure 2. Frequency of correct responses from condition peripheral-peripheral (top) and free-gaze (bottom) for young and older adults, as a function of distance between clusters. Symbols represent across-subject means, and error bars standard deviations.

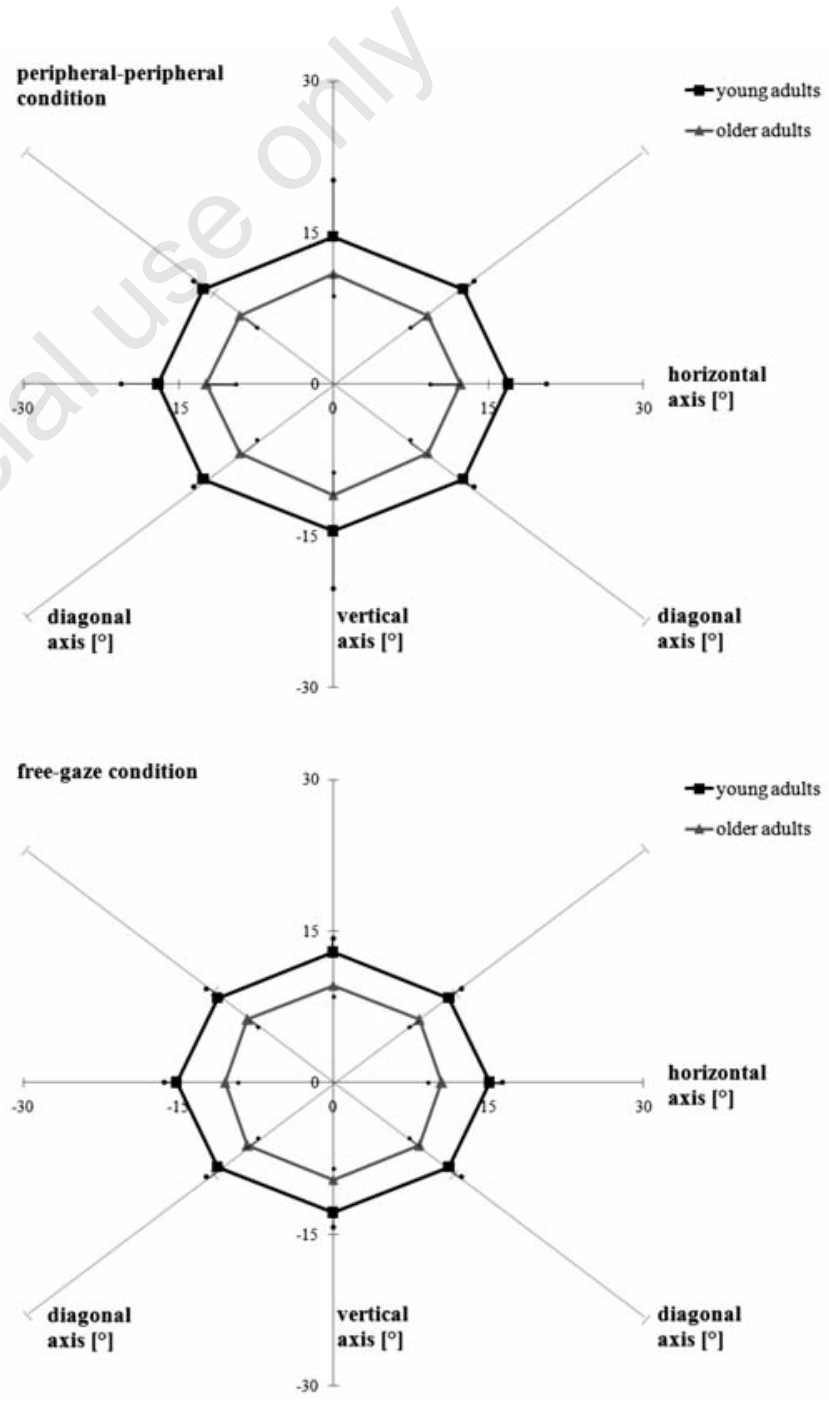

Figure 3. Size of the attention window, determined by the $75 \%$ correct criterion, for young and older adults, as a function of cluster distance. The top graphs show data from the peripheralperipheral, and the bottom graphs those from the free-gaze condition. Since each cluster pair was presented symmetrically about the center, the data are also symmetrical. Symbols represent across-subject means, and error bars standard deviations. 
we found a significant main effect of age but no significant interaction. Figure 2 illustrates that the difference between age groups existed already at the smallest cluster distance, increasing only slightly (and non-significantly) at larger distances. Retaining the metaphor of an attention window, our data suggest that this window does not shrink in old age, but rather becomes blurry. This again leads to practical implications. Since attentive processing is needed in everyday life not only in the far but also in the near periphery, training programs should include not only large, but also small cluster distances.

\section{References}

1. Kosnik W, Winslow L, Kline D, et al. Visual changes in daily life throughout adulthood. J Gerontol Psychol Sci 1988;43:6370.

2. Danner DD, Snowdon DA, Friesen WV. Positive emotions in early life and longevity: findings from the nun study. J Pers Soc Psychol 2001;80:804-13.

3. Sekuler AB, Bennett PJ, Mamelak M. Effects of aging on the useful field of view. Exp Aging Res 2000;26:103-20.

4. Shinar D, Schieber F. Visual requirements for safety and mobility of older drivers. Hum Factors 1991;33:507-19.

5. Crassini B, Brown B, Bowman K. Age related changes in contrast sensitivity in central and peripheral retina. Perception 1988;17:315-32.

6. Owsley C, Ball K, Keeton D. Relationship between visual sensitivity and target localization in older adults. Vision Res 1995;35: 579-87.

7. Owsley C, Ball K, Sloane ME, et al. Visual/cognitive correlates of vehicle accidents in older drivers. Psychol Aging 1991; 6:403-15.

8. Coeckelbergh TR, Cornelissen FW, Brouwer WH, Kooijman AC. Age-related changes in the functional visual field: fur- ther evidence for an inverse age $\mathrm{x}$ eccentricity effect. J Gerontol Psychol Sci 2004;59;11-8.

9. Edwards JD, Ross LA, Wadley VG, et al. The useful field of view test: normative data for older adults. Arch Clin Neuropsych 2006; 21:275-86.

10. Myers RS, Ball KK, Kalina TD, et al. Relation of useful field of view and other screening tests to on-road driving performance. Percept Motor Skill 2000;91: 279-90.

11. Sanders A. Some aspects of the selective process in the functional visual field. Ergonomics 1970;13:101-17.

12. Sekuler R, Ball K. Visual localization: age and practice. J Opt Soc Am 1986;3:864-7.

13. Ball K, Owsley C, Beard B. Clinical visual perimetry underestimates peripheral field problems in older adults. Clin Vis Sci 1990;5:113-25.

14. Mackworth NH. Stimulus density limits the useful field of view. In: Monty RA, Senders JW. Eye movements and psychological processes. Hillsdale, NJ: Lawrence Erlbaum Associates; 1976. pp. 307-321.

15. Ball K, Owsley C, Sloane M, et al. Visual attention problems as a predictor of vehicle crashes in older drivers. Invest Ophthalmol Vis Sci 1993;34:3110-23.

16. Ball K, Owsley $\mathrm{C}$. The useful field of view test: a new technique for evaluating agerelated declines in visual function. $\mathrm{J}$ Am Optom Assoc 1992;63:71-9.

17. Bono F, Oliveri RL, Zappia M, et al. Computerized analysis of eye movements as a function of age. Arch Gerontol Geriat 1996;22:261-9.

18. Irving EL, Steinbach MJ, Lillakas L, et al. Horizontal saccade dynamics across the human life span. Invest Ophthalmol Vis Sci 2006;47:2478-84.

19. Paquette C, Fung J. Old age affects gaze and postural coordination. Gait Posture 2011;33:227-32.

20. Moschner C, Baloh RW. Age-related changes in visual tracking. J Gerontol 1994;49:235-8.

21. Schneider W, Dumais ST, Shiffrin RM.
Automatic and control processing and attention. In: Parasuraman R, Davies DR. Varieties of attention. Orlando, FL: Academic Press; 1984. pp. 1-27.

22. Shiffrin RM, Schneider W. Controlled and automatic human information processing: II. Perceptual learning, automatic attending, and a general theory. Psychol Rev 1977;84:127-90.

23. Pöppel E, Harvey LOJ. Light-difference threshold and subjective brightness in the periphery of the visual field. PF 1973;36: 145-61.

24. McDowell JE, Dyckman KA, Austin BP, Clementz BA. Neurophysiology and neuroanatomy of reflexive and volitional saccades: evidence from studies of humans. Brain Cogn 2008;38:255-70.

25. Walker R, McSorley E. The parallel programming of voluntary and reflexive saccades. Vision Res 2006;46:2082-93.

26. Pashler H, Carrier M, Hoffman J. Saccadic eye movements and dual-task interference. Q J Exp Psychol 1993;46:51-82.

27. Mather JA, Putchat C. Parallel ocular and manual tracking responses to a continuously moving visual target. J Mot Behav 1983;15:29-38.

28. Bremmer F, Kubischik M, Hoffmann KP, Krekelberg B. Neural dynamics of saccadic suppression. J Neurosci 2009;29:12374-83.

29. Diamond MR, Ross J, Morrone MC. Extraretinal control of saccadic suppression. J Neurosci 2000;20:3449-55.

30. Ball K, Beard B, Roenker D, et al. Visual search: age and practice. Invest Ophthalmol Vis Sci 1988;29:448.

31. Seiple W, Szlyk JP, Yang S, Holopigian K. Age-related functional field losses are not eccentricity dependent. Vision Res 1996; $36: 1859-66$.

32. Ball K, Roenker D, Rizzo M, McGehee D. Cognitive training to improve driving competence. Gerontologist 2000;40:41.

33. Roenker DL, Cissel GM, Ball KK, et al. The effects of speed of processing and driving simulator training on driving performance. Hum Factors 2003;45:218-33. 